\title{
Isomechanical Groups in Molecular Crystals and Role of Aromatic Interactions
}

\author{
Benjamin P. A. Gabriele ${ }^{\mathrm{a}}$, Craig J. Williams ${ }^{\mathrm{b}}$, Matthias Eckhard Lauer ${ }^{\mathrm{c}}$, Brian Derby, \\ Aurora J. Cruz-Cabeza ${ }^{\text {a* }}$ \\ ${ }^{a}$ Department of Chemical Engineering and Analytical Science, University of Manchester, UK. \\ ${ }^{b}$ Department of Materials, University of Manchester, UK. \\ ${ }^{c}$ Roche Innovation Center Basel, Basel, Switzerland \\ *Email: aurora.cruzcabeza@manchester.ac.uk
}

\begin{abstract}
This Electronic Supplementary Information (ESI) includes the trapezoid load functions used for the nanoindentation experiments, the DSC results obtained on all the acids and their extracted melting point, the histograms of the pop-ins obtained during indentation of benzoic acid and p-toluic acid, and the computed Periodic Bond Chains (PBCs) obtained.
\end{abstract}

\section{S1. Experimental Methods}

Nanoindentation and pop-ins analysis. Experiments were carried out on 7 to 10 single crystals per molecule studied (9 indents per crystal) using a Hysitron Ti950 Triboindenter (Hysitron/Bruker) equipped with a diamond Berkovich probe. Indentations were conducted in either load-control or displacement-control mode using a trapezoid load function. We first investigated the optimal maximum displacement/load as defined in a previous work, so the modulus and hardness measured do not vary for further increase of decrease of $P_{M A X}$ or $h_{M A X}{ }^{1}$ The trapezoid load functions used were hence adapted for each compound studied. Additionally, pop-ins were measured and compiled from the 90 indentation curves obtained by indenting benzoic acid and $p$-toluic acid single crystals.

DSC. Melting points were assessed via Differential Scanning Calorimetry (DSC, DSC2500, TA Instruments). Between 3 and 8 single crystals, depending on the crystals size, were placed into DSC pans (Tzero Pan T181128, TA Instruments). Three independents measurements were made per compound, with one unique heating cycle per measurement from $30^{\circ} \mathrm{C}$ up to $150-300^{\circ} \mathrm{C}$ depending on the expected melting point.

Calculation of Lattice energiesEnergies were calculated using Material Studio 2019 (Dassault Systèmes, BIOVIA). ${ }^{2}$ The energy of the crystal was calculated by relaxing the atoms positions using the forcefield COMPASSII (with its own forcefield charges) while keeping the unit cell parameters constants. The crystal energy was then normalised by the number of molecules in the unit-cell $\left(U_{C R Y S T A L}\right)$. A separate simulation was done in which a single molecule of interest was fully optimised in the gas phase $\left(\mathrm{E}_{\mathrm{GAS}}\right)$. The lattice energy $U_{L A T T}$ was calculated for each compound as $U_{L A T T}=U_{C R Y S T A L}-U_{G A S}$. PBCs were calculated using the same energy model and a cut off of $-13 \mathrm{~kJ} / \mathrm{mol}$ for visualisation. 


\section{S2. Nanoindentation - Load function}

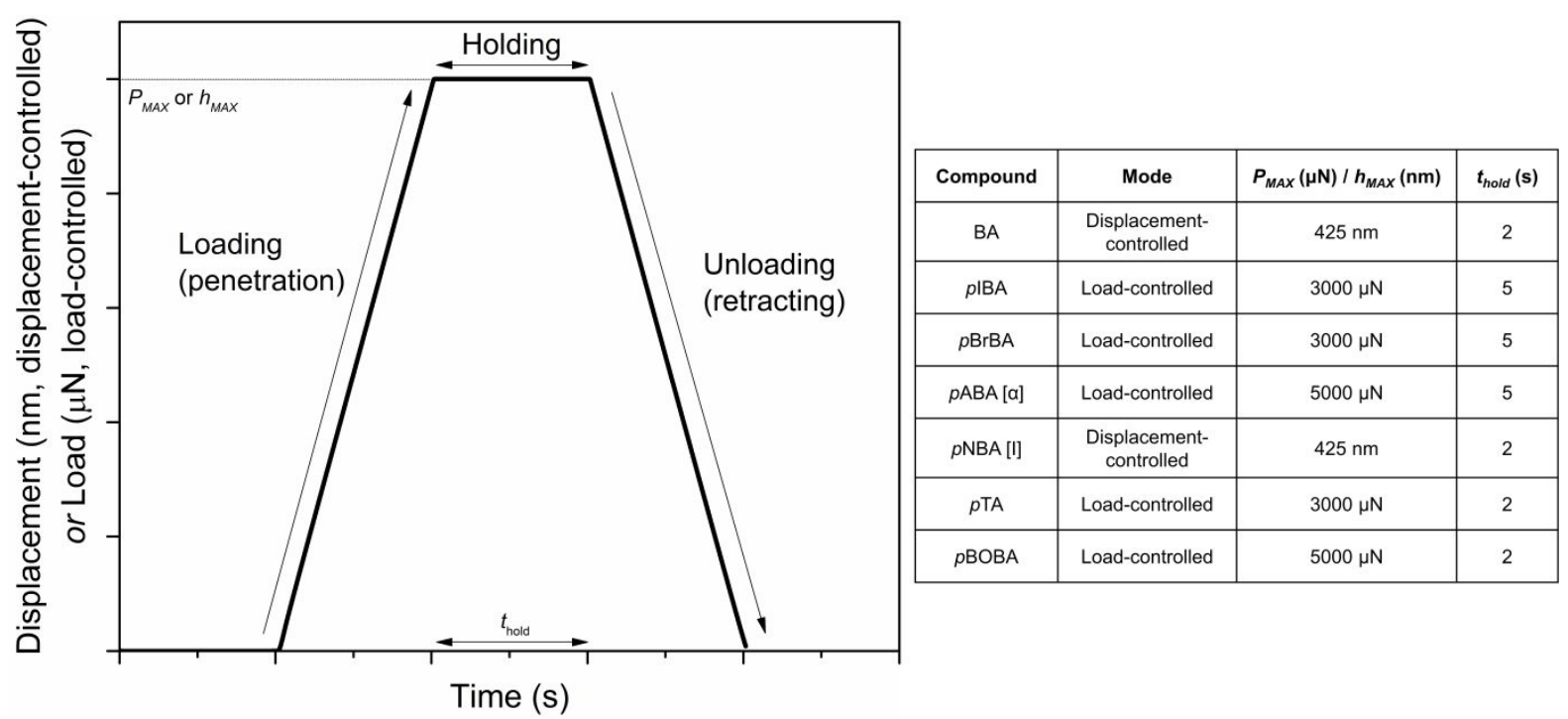

Figure S2.1: The trapezoid load function used is presented here along with the different parameters, namely the mode used (load-controlled or displacement-controlled), the maximum load $P_{M A X}$ or maximum displacement $h_{M A X}$ used and the holding time used to reduce viscous effects.

\section{S3. DSC results}

DSC results obtained on benzoic acid and its six derivatives are plotted in Figure S3.1. Melting points were extracted as the on-set melting temperature using the software TRIOS (Version 5.0.0, TA Instruments). The melting events were clearly identified for all compounds as a unique endothermic peak.

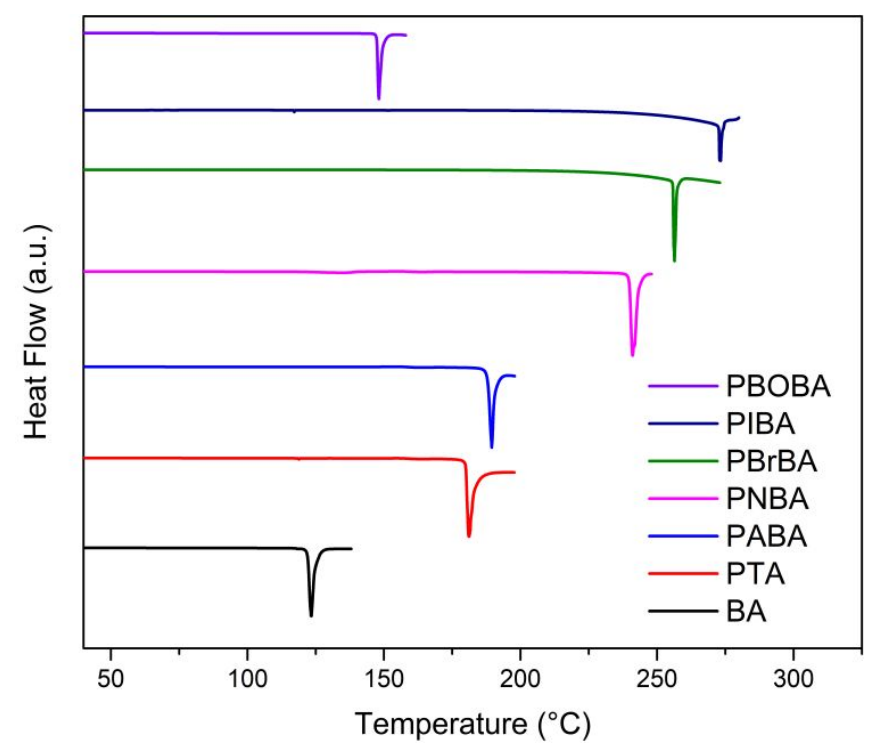

Figure S3.1: DSC measurements obtained on single crystals (3 to 8 crystals depending on their size) of benzoic acid and six of its derivatives. Each cycle showed for every compound shows a clear, unique endothermic peak corresponding to the melting point. 


\section{S4. Pop-ins results}

When indenting molecular single crystals, pop-ins intensities have been reported to match crystallographic data such as unit cell length or interplanar spacing, due to the gliding of crystal planes along such orientation. ${ }^{3-5}$ Only a few pop-ins occurred per curve for benzoic acid, which hindered greatly such analysis. The highest repetition of pop-ins intensities measured were centred around $2.15 \mathrm{~nm}$, which could match notably twice $d(002)=1.0884$ $\mathrm{nm}$, with (002) is the face indented. Still, many pop-ins have intensities which are not integer of $d(002)$, so such interpretation should be made with care. These results are shown as histograms in Figure S4.1a. In the case of $p$ TA, pop-ins were better identified to match crystallographic data when using the refcode PTOLIC01 rather than PTOLIC. Using PTOLIC01, the corresponding face we indented becomes (-111) (PTOLIC and PTOLIC01 do not have the same unit cell parameters). Integers of the interplanar spacing $d(1-1-1)=$ $0.50395 \mathrm{~nm}$ were found to match several of the highest pop-in intensities counts centred around $6.55 \mathrm{~nm}, 7.55 \mathrm{~nm}, 8.05 \mathrm{~nm}$ and $8.5 \mathrm{~nm}$ (see Figure S4.1b). Such correlation fails when comparing the pop-ins intensities with crystallographic data from PTOLIC, although this CSD refcode was determined at room temperature whereas PTOLIC01 was determined at a temperature of $100 \mathrm{~K}$.

a) Benzoic Acid

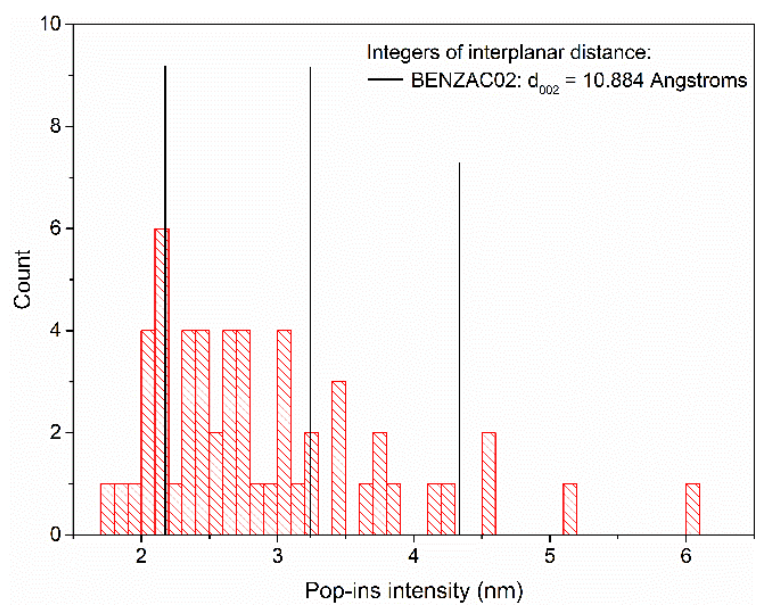

b) $p$-Toluic Acid

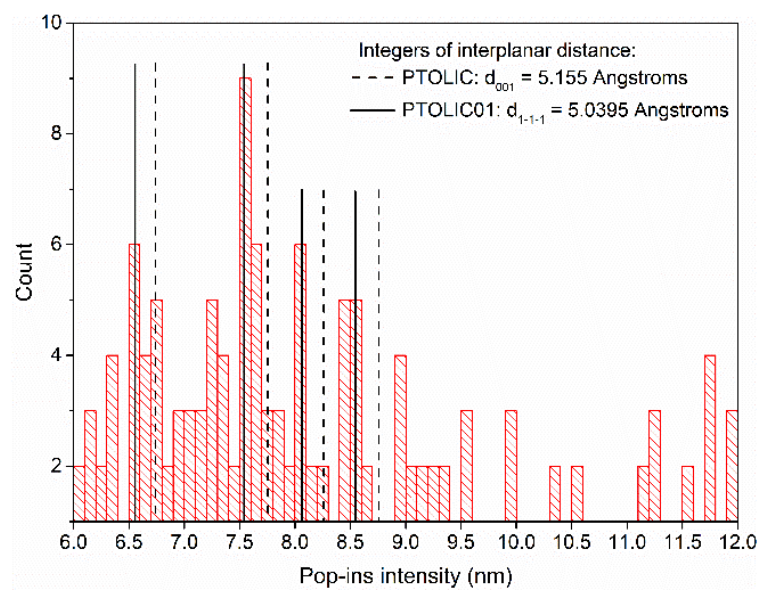

Figure S4.1: Histograms of the count of the pop-ins intensities across the 90 indentation measurements for each study, on (a) BA and (b) p TA. Correlation with the interplanar distance of the respective plane indented is indicated as vertical black line. For $p$ TA, a better correlation was found using the refcode PTOLIC016 ${ }^{6}$, although this CSD refcode does not corresponds to a crystal structure measured at room temperature but at 100 Kelvins. 


\section{S5. Periodic Bond Chains results}

Periodic bond chains (PBCs) were computed using Material Studio: from the associated .cif files available within the CSD, a geometry optimisation was carried out, followed by the calculation of the intermolecular energy. A cut-off of $-13 \mathrm{~kJ} / \mathrm{mol}$ was applied. The hydrogen atoms in the .cif files associated with the CSD refcodes PTOLIC and BRBZAP (respectively PTA and PBrBA) were correctly replaced before the computation of the corresponding energy framework. Figure S5.1 shows the results for BA, $p$ TA, $p$ ABA, $p$ NBA, $p$ IBA and $p \mathrm{BrBA}$. This results in a $1 \mathrm{D}$ energy network (2D for $p \mathrm{TA}$ and $p \mathrm{ABA}$ ). For all compounds, two consecutive planes as seen in Figure S5.1 are only bonded together by intermolecular interaction of energy lower than the cut-off, i.e. very weak interactions. We conclude here that these six compounds all have an energy framework that enables plastic deformation through easy slip of planes, as compared to the 3D energy framework associated with $p$ butoxybenzoic acid as shown in Figure S5.2. Such 3D network contrasts with those observed for the other acids thus mechanical properties measured by nanoindentation on $p \mathrm{BOBA}$ crystals were stiffer and harder than expected.

a) $\mathrm{BA}$

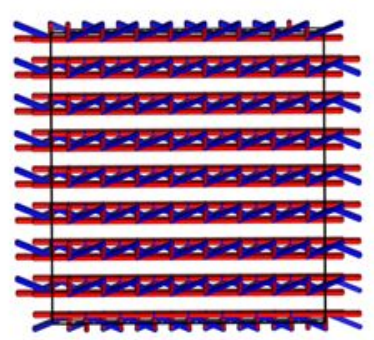

c) PABA

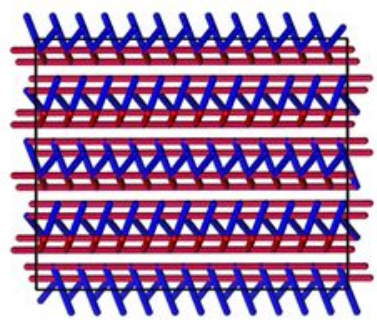

e) PIBA

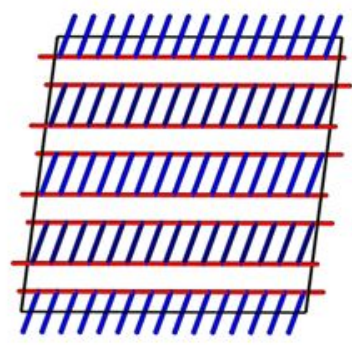

b) PTA
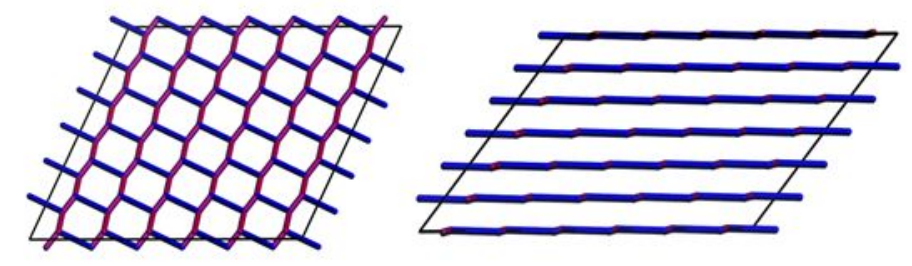

d) PNBA

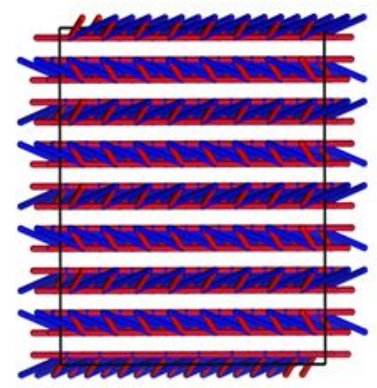

f) $\mathrm{PBrBA}$

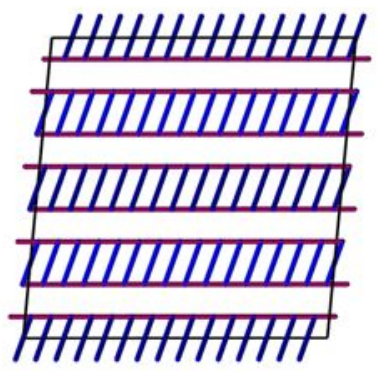

Figure S5.1: Energy framework of benzoic acid and five of its derivatives viewed along the indentation axis. An energy cut-off of $-13 \mathrm{~kJ} / \mathrm{mol}$ has been applied. The energy framework corresponding to PTA crystal packing is shown both along the indentation axis ( $b$, left) and perpendicular to it (b, right). 
a)

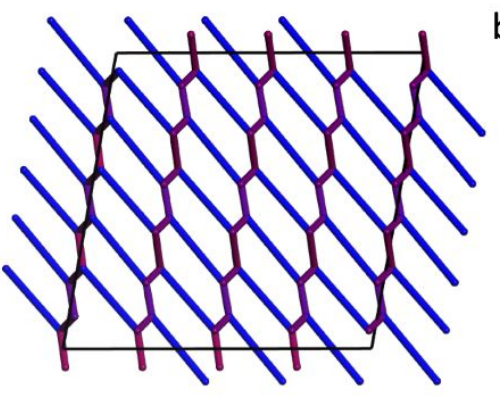

b)

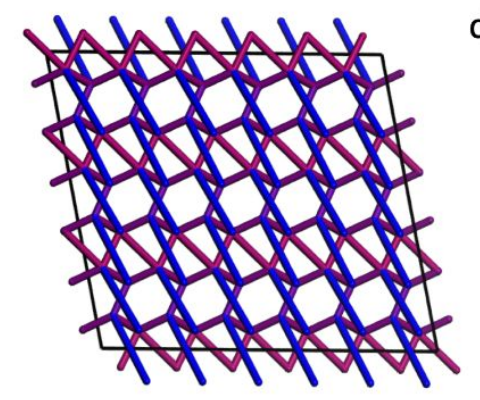

c)

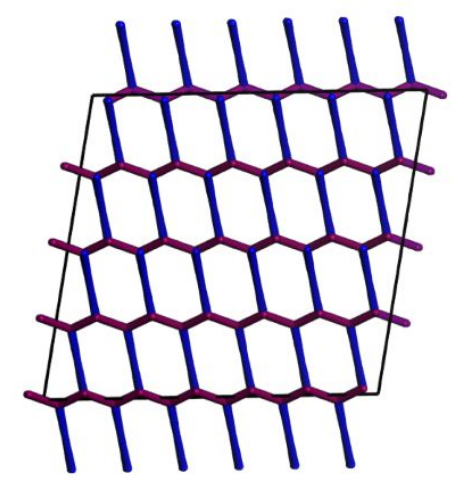

Figure S5.2: Energy framework of $p$-Butoxybenzoic acid viewed along the (a) a-axis (b) the indentation axis, the b-axis, and (c) the c-axis (CSD refcode: BUXBZA02). An energy cut-off of $-13 \mathrm{~kJ} / \mathrm{mol}$ has been applied. The framework shows a considerably more 3D bonded network with no evident slip plane. 


\section{References}

(1) Gabriele, B. P. A.; Williams, C. J.; Lauer, M. E.; Derby, B.; Cruz-Cabeza, A. J. Nanoindentation of Molecular Crystals: Lessons Learnt from Aspirin. Cryst. Growth Des. 2020, DOI: $10.1021 /$ acs.cgd.0c00635.

(2) Dassault Systèmes BIOVIA, Materials Studio, Release 2019, San Diego: Dassault Systèmes. 2019.

(3) Kiran, M. S. R. N.; Varughese, S.; Reddy, C. M.; Ramamurty, U.; Desiraju, G. R. Mechanical Anisotropy in Crystalline Saccharin: Nanoindentation Studies. Cryst. Growth Des. 2010, 10, 4650-4655.

(4) Varughese, S.; Kiran, M. S. R. N.; Solanko, K. A.; Bond, A. D.; Ramamurty, U.; Desiraju, G. R. Interaction Anisotropy and Shear Instability of Aspirin Polymorphs Established by Nanoindentation. Chem. Sci. 2011, 2, 2236-2242.

(5) Mishra, M. K.; Ramamurty, U.; Desiraju, G. R. Solid Solution Hardening of Molecular Crystals: Tautomeric Polymorphs of Omeprazole. J. Am. Chem. Soc. 2015, 137, 1794-1797.

(6) Hathwar, V. R.; Thakur, T. S.; Row, T. N. G.; Desiraju, G. R. Transferability of Multipole Charge Density Parameters for Supramolecular Synthons: A New Tool for Quantitative Crystal Engineering. Cryst. Growth Des. 2011, 11, 616-623. 